\section{Comparison of trabeculectomy and Ex-PRESS implantation in fellow eyes of the same patient: a prospective, randomised study}

E Dahan', GJ Ben Simon² and A Lafuma ${ }^{3}$

\begin{abstract}
Purpose To compare intraocular pressure (IOP) over time after standard trabeculectomy vs Ex-PRESS implantation in patients with bilateral primary open-angle glaucoma (POAG).

Design Prospective, randomised study. Patients and methods This study included adult patients with bilateral POAG necessitating surgery. Each patient underwent trabeculectomy in one eye and Ex-PRESS implantation under a scleral flap in the other eye according to randomised contralateral allocations. Efficacy was assessed by IOP values and success rates (IOP threshold and/or need for topical glaucoma medication) during 30 months. Statistical analysis included Generalised Estimate Equation and Cox Survival models, and paired $t$-tests.

Results Thirty eyes of 15 patients were studied for a mean of 23.6 months (SD, \pm 6.9). At the last follow-up visit, mean pre-operative IOP decreased from $31.1( \pm 14.2)$ to $16.2( \pm 1.5)$ $\mathrm{mm} \mathrm{Hg}$ after trabeculectomy, and from 28.1 $( \pm 9.0)$ to $15.7( \pm 1.8) \mathrm{mm} \mathrm{Hg}$ after Ex-PRESS implantation $(P=0.001)$. The mean number of anti-glaucoma medicines prescribed at the last follow-up decreased from 3.7 pre-operatively (both groups) to 0.9 after trabeculectomy vs 0.3 after Ex-PRESS implantation $(P=\mathbf{0 . 0 0 1})$. Complete success rates $(5<\mathrm{IOP}<18 \mathrm{~mm} \mathrm{Hg}$ without medications) were higher with Ex-PRESS compared with trabeculectomy $(P=0.0024)$. Postoperative complications were more frequent after trabeculectomy (33\%) compared with Ex-PRESS (20\%), with four trabeculectomy eyes $(27 \%)$ needing
\end{abstract}

postoperative interventions, compared with none with Ex-PRESS.

Conclusions Trabeculectomy and Ex-PRESS implantation provided similar IOP control, but the Ex-PRESS group had a lower rate of complications, fewer postoperative interventions, and needed less glaucoma medications.

Eye (2012) 26, 703-710; doi:10.1038/eye.2012.13; published online 17 February 2012

Keywords: trabeculectomy; Ex-PRESS; primary open-angle glaucoma

\section{Introduction}

Trabeculectomy is the most commonly performed incisional procedure for intraocular pressure (IOP) reduction in glaucoma patients. Its success rate and complications are well established. ${ }^{1,2}$ Short-term complications after trabeculectomy include anterior chamber shallowing, hypotony, and choroidal detachment. Long-term complications include bleb leaks, blebitis/endophthalmitis, overhanging blebs, and bleb failure. These potential complications threaten vision thereby demonstrating the need for safer surgical procedures to manage glaucoma. In recent years several alternative procedures have been evaluated and compared to trabeculectomy. ${ }^{1-5}$

The Ex-PRESS glaucoma filtration device (Alcon Laboratories, Fort Worth, TX, USA) was developed as an alternative to trabeculectomy, and shows similar IOP reduction with fewer complications. Its original design required insertion under a conjunctival flap through the
'Department of Ophthalmology, University of the Witwatersrand, Johannesburg, South Africa

${ }^{2}$ Goldschleger Eye Institute, Sheba Medical Center, Tel Hashomer, Israel

${ }^{3}$ Cemka-Eval, Bourg la Reine, France

Correspondence: GJ Ben Simon, Goldschleger Eye Institute, Sheba Medical Center, Tel Hashomer, Ramat Gan 52621, Israel Tel: + 9723 5302874; Fax: + 97235302822 . E-mail: guybensimon@ gmail.com

Received: 5 May 2011 Accepted in revised form: 4 January 2012 Published online: 17 February 2012

This study was reported in part at the Israel Glaucoma Society Meeting, March 2008. 
full scleral thickness. This procedure led to unacceptable hypotony rates in the immediate postoperative stage and medium-term erosion of the device through the conjunctiva. ${ }^{6-12}$ Dahan and Carmichael ${ }^{13}$ addressed these issues by placing the device under a partial-thickness scleral flap. The original sub-conjunctival placement has now been completely abandoned for a safer implantation under a scleral flap. ${ }^{13-20}$ The latter procedure closely resembles trabeculectomy, but offers the advantages of a standard (stented) sub-scleral outflow route that obviates sclerostomy and peripheral iridectomy.

Until now, data comparing safety and efficacy of standard trabeculectomy vs Ex-PRESS implantation under a scleral flap were mostly retrospective and the procedures have been evaluated in eyes with diverse types of glaucoma. ${ }^{14,15,18,20}$ In this report we describe the results of a prospective, randomised study in patients with bilateral primary open-angle glaucoma (POAG) only, where both procedures (one per eye) were compared in fellow eyes of the same patient.

\section{Patients and methods}

\section{Study design and surgical technique}

Patients with bilateral POAG underwent standard trabeculectomy in one eye and Ex-PRESS implantation under a scleral flap in the other eye, according to a prospective and randomised design.

Eligible patients were at least 18 years of age and presented with medically uncontrolled POAG requiring bilateral incisional surgery for IOP reduction. Patients with prior cataract operation or failed filtration surgery in either eye were eligible if surgery took place at least 3 months prior to enrolment. Patients meeting any of the following criteria were excluded: any form of glaucoma other than POAG; history of active uveitis; and any ocular abnormality that would preclude accurate IOP assessment.

The study was conducted in accordance with the tenets of the Declaration of Helsinki. All participating patients provided written informed consent. The study was approved by the Ethics Committee of the Medical School at the University of the Witwatersrand, Johannesburg, South Africa. The trial is registered at http:/ / www.clinicaltrials.gov (unique registration number: NCT00698438).

After consent was given, patients' preoperative baseline data were collected, including demographics, ocular history, visual acuity, applanation tonometry, slit-lamp biomicroscopy, and ophthalmoscopy. Randomisation of contralateral operations was achieved by opening an envelope in which the procedure (trabeculectomy or Ex-PRESS implantation) that would be applied to the first eye was stated, thereby determining the procedure in the other eye.

After sub-tenonian local anaesthesia, surgery was performed by one experienced surgeon (ED), for consistency, using a standardised technique for both procedures. In all cases a $25 \mathrm{G}$ anterior chamber maintainer (ACM), connected to a bottle of balanced salt solution (BSS), was inserted into the limbus at the lower temporal quadrant to maintain positive IOP throughout the operation. A fornix-based conjunctival flap was created with a relaxing incision on one side. Next, a $50 \%$ thickness trapezoidal scleral flap $(5 \times 5 \times 1 \mathrm{~mm})$ was constructed and advanced anteriorly into the clear cornea. Then, for all patients a wound-modulating agent (mitomycin C $0.05 \%$ ) was applied to the scleral bed for $1 \mathrm{~min}$. For trabeculectomy a $1.5 \times 1-\mathrm{mm}$ posterior lip sclerectomy was fashioned with a 15 degree surgical blade under the scleral flap, at the grey limbal zone between the white sclera and the clear cornea, and a peripheral iridectomy was created. For eyes randomised to Ex-PRESS, the Ex-PRESS X200 model, designed for implantation under a scleral flap, was selected. This model slightly differs from the original Ex-PRESS R50 version designed for implantation under the conjunctiva because it has a slitted flange, total $2.4 \mathrm{~mm}$ length, a rounded tip, a square body, and a $200-\mu \mathrm{m}$ micron lumen. Ex-PRESS X200 was implanted with a 23-G needle held parallel to the iris, to produce a pre-incision under the scleral flap, through the grey zone, and into the anterior chamber. Mobility of the Ex-PRESS device on its introducer was confirmed before the device was implanted through the needle track. Closure was identical with both procedures. The scleral flap was closed with five interrupted 10-0 nylon sutures (one suture at the apex and two at each side of the scleral flap). Water tightness was verified by allowing the BSS fluid to ooze out very slightly near the apex of the trapezoidal scleral flap with the bottle positioned $50 \mathrm{~cm}$ above the patient's head. The conjunctiva was closed with two 10-0 nylon sutures tacking down both ends of the peritomy. In all cases, at the end of surgery the anterior chamber was filled with standard-viscosity, viscoelastic material (Healon, Provisc) via the paracentesis port created for the ACM. Following surgery, eyes were initially treated with a steroid/ antibiotic combination ( $\times 4$ daily) for 2 weeks and thereafter with a non-steroidal, anti-inflammatory drug (Diclofenac $0.1 \% \times 3$ daily) for 8 weeks. During the early postoperative period, hypertony ( $\geq 27 \mathrm{~mm} \mathrm{Hg}$ ) was treated with $\mathrm{A} / \mathrm{C}$ decompression using the ACM paracentesis port if it occurred during the first week postoperatively. Scleral flap suture cutting was performed if hypertony occurred later. Patients were evaluated after surgery on days 1 and 7, at months 1, 3, 6, 
9 and 12, and at 6-monthly intervals thereafter. The following items were assessed, at each visit: surgical complications, IOP, visual acuity, and number of medicines needed to achieve adequate IOP control.

\section{Data analysis}

Power

The study sample size $(n=30$, ie, 15 eyes with each procedure) provided $96 \%$ power to detect an average IOP difference of $2.0 \mathrm{~mm} \mathrm{Hg}$ between eyes (average IOP, $14( \pm 2.5)$ vs $16.5 \mathrm{~mm} \mathrm{Hg}( \pm 2.5)$, with a follow-up correlation of 0.25 between eyes that underwent Ex-PRESS implantation $v$ s trabeculectomy), based on the planned number of measurements (ie, 7) starting at 1 month after surgery. The study had $84 \%$ power to detect an average difference of $1.6 \mathrm{~mm} \mathrm{Hg}$ and $79 \%$ power to detect an average IOP difference of $1.5 \mathrm{~mm} \mathrm{Hg}$. Power calculations were based on a type-I $\alpha$ error of 0.05 and two-sided tests.

\section{Statistical analysis}

Wilcoxon matched-pairs $t$-test (within patients) was used to compare pre-operative and final IOP values, and the number of glaucoma medicines.

A Generalised Estimating Equation (GEE) model $^{21}$ was used to analyse the time trend of the average IOP difference between eyes receiving randomised treatments, from 1 month after surgery to the end of the follow-up period (30 months). All available IOP follow-up data at days 1 and 7, and months 1, 3, 6, 9, 12, 18,24 and 30 were analysed. The study was specifically designed to satisfy such a model. GEE models allow individual IOP correlations over time, as these may differ between patients. Also, fractional polynomials ${ }^{22,23}$ were incorporated to adjust for postoperative time effects. Fractional polynomials enable a flexible strategy, modelling both linear and non-linear effects arising with continuous variables (in the present case, effects of time from 1 month following surgery). The estimated average IOP difference between procedures over time was then plotted with its $95 \%$ confidence interval (CI). The power of both the study and the GEE model were estimated using Stata version 11 (Stata Corp., College Station, TX, USA).

A Cox survival model was used to compare clinical failure between procedures. Complete success was defined as IOP $>5 \mathrm{~mm} \mathrm{Hg}$ and $<18 \mathrm{~mm} \mathrm{Hg}$ without glaucoma medication. Qualified success was defined as IOP $>5 \mathrm{~mm} \mathrm{Hg}$ and $<18 \mathrm{~mm} \mathrm{Hg}$ with or without glaucoma medication. To take into account the correlation between an individual patient's eyes, a robust estimate of the variance was calculated using a JackKnife estimator, as recommended by Therneau and
Grambsch $^{24}$ and implemented in the ' $R$ survival package' (R version 2.11.1, Survival Package Version 2.36-2). ${ }^{25,26}$ Cross-tabulations using MacNemar's $\chi^{2}$-test were used to calculate the proportion of complications with each procedure (trabeculectomy vs Ex-PRESS). Other statistical tests included Wilcoxon matched paired $t$-test, Fisher's exact test, and Kaplan-Meier survival analyses.

\section{Results}

Thirty eyes of 15 patients with bilateral POAG were included in the study. They comprised 10 males and 5 females with mean age $65.4( \pm 13.7)$ years. Six patients were black, 5 Indian, 3 Caucasian, and 1 mixed. All patients suffered from advanced bilateral POAG. Baseline glaucoma severity was similar in each eye pair, with a mean cup disk ratio $\geq 0.85$ for both groups. Pre-operatively, mean IOP was $29.6 \mathrm{~mm} \mathrm{Hg}( \pm 11.8)$ for all 30 eyes, distributed as $31.1 \mathrm{~mm} \mathrm{Hg}( \pm 14.2)$ for 15 trabeculectomy eyes and $28.1 \mathrm{~mm} \mathrm{Hg}( \pm 9.0)$ for 15 Ex-PRESS eyes $(P=0.484)$, after randomisation. Glaucoma medication prior to surgery averaged 3.7 $( \pm 0.7)$ medical entities administered to both eyes. The 15 eyes of each group included 10 glaucoma surgery failures because 10 patients had bilateral previously failed surgery. All 15 patients were followed-up for 1 year after surgery. One patient died 13 months after surgery and two patients were subsequently lost to follow-up. All data available for these patients (ie, up to 1 year) are included in the analyses.

At the last follow-up, mean IOP values decreased significantly (paired $t$-test $P<0.001$ ) from their preoperative levels, that is, trabeculectomy, from 31.1 ( \pm 14.2$) \mathrm{mm} \mathrm{Hg}$ to $16.2( \pm 1.5) \mathrm{mm} \mathrm{Hg}$, and Ex-PRESS, from $28.1( \pm 9.0) \mathrm{mm} \mathrm{Hg}$ to $15.7( \pm 1.8) \mathrm{mm} \mathrm{Hg}$, thus demonstrating the efficacy of both surgical techniques. Similarly, at the last follow-up, mean anti-glaucoma medicine consumption decreased significantly (paired t-test $P<0.001$ ), from 3.7 ( \pm 0.5$)$ pre-operatively, to 0.9 $( \pm 1.0)$ after trabeculectomy and $0.3( \pm 0.5)$ after Ex-PRESS. Results on evolution of IOP and number of medications in both groups are described in Figure 1.

Figure 2 shows the GEE model time trend of the mean IOP difference between eyes randomised to Ex-PRESS implantation compared with trabeculectomy, from 1 month after surgery to the end of the 30-month follow-up period. The horizontal dashed line indicates no difference in IOP between procedures. The solid line with its shaded 95\% CI represents a significant mean IOP difference between eyes with Ex-PRESS compared with trabeculectomy, from 3 to 24 months after surgery and no difference at 30 months.

Kaplan-Meier survival analysis curves for complete and qualified successes are shown in Figures 3 and 4. 


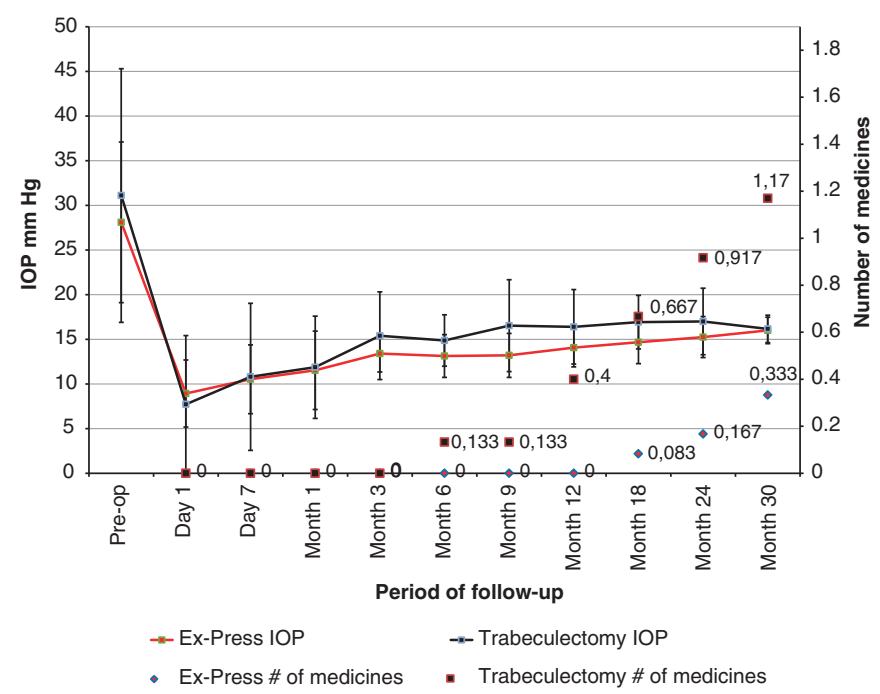

Figure 1 IOP ( $\mathrm{mm} \mathrm{Hg}$ ) and number of glaucoma medicines over time according to surgical technique.

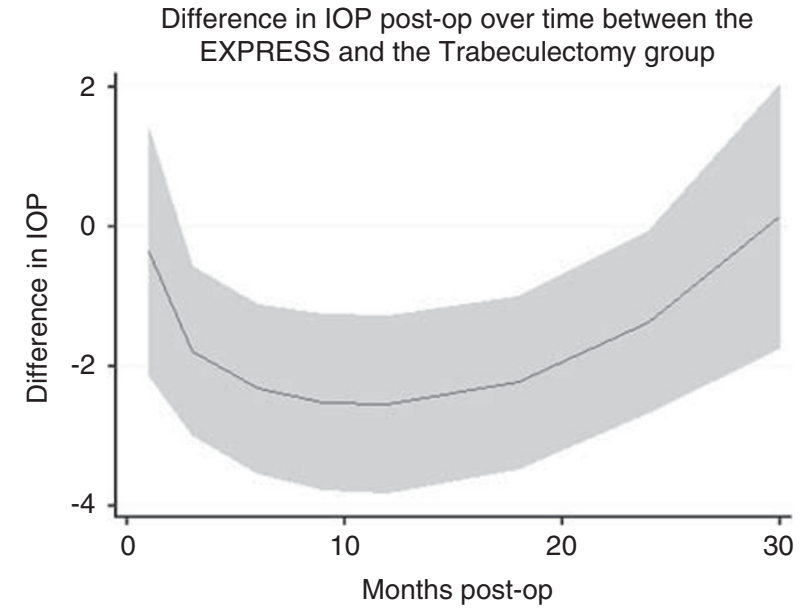

Figure 2 Time trend of the average difference in IOP between Ex-PRESS $v$ s trabeculectomy eyes from 1 month after surgery to the end of follow-up (30 months). Note: For all time points in which the 95\% CI (grey shaded zone) of the average difference in IOP between Ex-PRESS $v$ s trabeculectomy eyes lies below the dashed line (difference in $\mathrm{IOP}=0$ ), Ex-PRESS eyes had a significantly lower (better) IOP.

Figure 3 depicts complete success probabilities after both trabeculectomy and Ex-PRESS implantation, with success defined as IOP $>5 \mathrm{~mm} \mathrm{Hg}$ and $<18 \mathrm{~mm} \mathrm{Hg}$ on two consecutive visits (or at the last visit) without glaucoma medication. A higher probability of success followed Ex-PRESS implantation (hazard ratio $=0.27$ $\left.\left(\mathrm{CI}_{95 \%}=(0.12 ; 0.60)\right)\right)$ compared with standard trabeculectomy $(P=0.0019)$.

Figure 4 shows probabilities of qualified success (with and without glaucoma medication), yielding a hazard

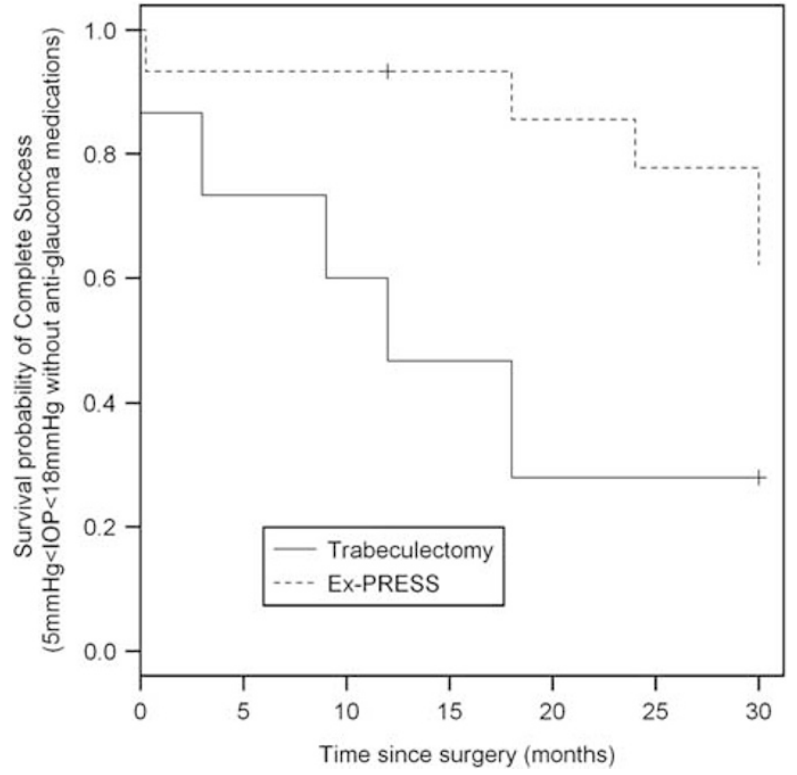

Figure 3 Survival curve with complete success defined as $5<\mathrm{IOP}<18 \mathrm{~mm} \mathrm{Hg}$ without glaucoma medication $(P=0.0024)$.

ratio of $0.21\left(\mathrm{CI}_{95 \%}=(0.06 ; 0.71)\right)$ in favour of Ex-PRESS, compared with standard trabeculectomy $(P=0.0124)$.

Patients with at least one postoperative complication during any visit are summarised in Table 1.

Complications were generally mild although more frequent after trabeculectomy (33\%) than after Ex-PRESS implantations $(20 \%)(P=0.05$, Fisher's exact test).

Further surgical interventions were needed for four eyes $(27 \%)$ in the trabeculectomy group, only $(P=0.0009$, Fisher's exact test). In addition, one trabeculectomy eye underwent hyphema and an Ex-PRESS eye a wound 


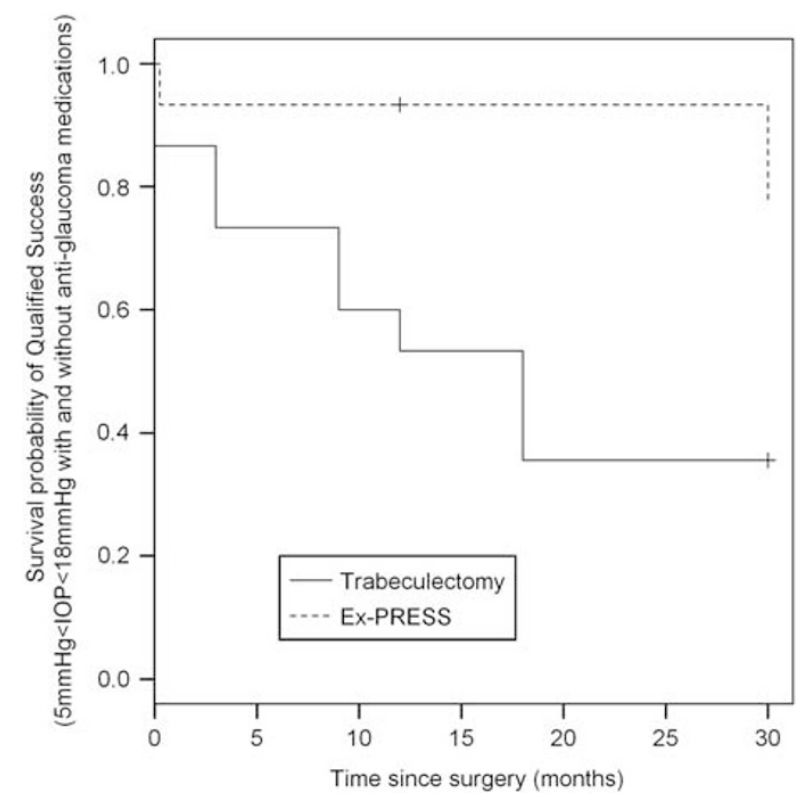

Figure 4 Survival curve with qualified success defined as $5<\mathrm{IOP}<18 \mathrm{~mm} \mathrm{Hg}$ with and without glaucoma medication $(P=0.0124)$.

Table 1 Number of complications and postoperative interventions occurring at least once per patient $(n=15)$

\begin{tabular}{lll}
\hline Complications & $\begin{array}{c}\text { Trabecule } \\
\text { ctomy-PRESS } \\
\text { no. }(\%)\end{array}$ & \\
& & no. $(\%)$ \\
& $3(20 \%)$ & $2(13 \%)$ \\
Shallow anterior chamber & $5(33 \%)$ & $1(7 \%)$ \\
Hypotony $(\leq 4 \mathrm{~mm} \mathrm{Hg})$ & $3(20 \%)$ & $0(0 \%)$ \\
Hypertony $(\geq 27 \mathrm{~mm} \mathrm{Hg})$ & $1(7 \%)$ & $0(0 \%)$ \\
Hyphema & $0(0 \%$ & $1(7 \%)$ \\
Wound leak & $1(7 \%)$ & $0(0 \%)$ \\
High avascular bleb & $5(33 \%)$ & $3(20 \%)$ \\
At least one complication per eye & & \\
$(P=0.05)$ & & \\
& $1(7 \%)$ & $0(0 \%)$ \\
Postoperative interventions & $2(13 \%)$ & $0(0 \%)$ \\
$\quad$ Anterior chamber reformation & $1(7 \%)$ & $0(0 \%)$ \\
Anterior chamber decompression & $4(27 \%)$ & $0(0 \%)$ \\
Bleb excision & & \\
At least one postoperative intervention & & \\
per eye $(P=0.0009)$ & & \\
\hline
\end{tabular}

leak; these mild complications both resolved spontaneously within a week.

Early in the postoperative course, hypotony $(\mathrm{IOP}<5 \mathrm{~mm} \mathrm{Hg}$ ) was more frequently observed after trabeculectomy $(33 \%)$ than after Ex-PRESS implantation (7\%). In all cases it resolved during the first month after either intervention. Total anterior chamber loss with iridocorneal touch or choroidal detachment was never encountered in either group. Two eyes required anterior chamber decompression for elevated IOP during the early postoperative course after trabeculectomy, but no eyes needed suture lysis. At the 1-year follow-up, one eye after trabeculectomy underwent bleb excision with advancement of healthy conjunctiva because of a high, avascular and very thin bleb. By contrast, no Ex-PRESS eye required any surgical intervention during the follow-up period.

During the follow-up period, no acceleration of cataract formation was encountered after both interventions and visual acuity remained unchanged relative to pre-operative values.

\section{Discussion}

Trabeculectomy has been the standard IOP-lowering procedure for nearly 40 years, but its potentially devastating sight-threatening complications have prompted calls for a better, safer operation. ${ }^{27}$ In recent years several new IOP-lowering procedures have been developed as alternatives to standard trabeculectomy. These include non-penetrating procedures such as deep sclerectomy with or without collagen implant, viscocanalostomy, and novel angle procedures. ${ }^{28}$ Also, glaucoma drainage devices have been developed and evaluated as primary surgical therapies for glaucoma. ${ }^{29}$

Numerous studies have reported on the biocompatibility, safety, and efficacy of the Ex-PRESS glaucoma filtration device during its evolution over the last decade. ${ }^{30-33}$ The device is essentially a trans-scleral miniature tube that delivers aqueous from the anterior chamber to the sub-scleral and sub-conjunctival spaces (similar to trabeculectomy). Ex-PRESS implantation under a scleral flap may be viewed as a compromise between trabeculectomy and non-penetrating glaucoma surgery on account of the standardised flow restriction imposed by the miniature shunt. The procedure, however, is more straightforward than trabeculectomy as it does not require sclerostomy or iridectomy. This also may reduce postoperative inflammation, which is desirable after glaucoma surgery. In rabbits, levels of transforming growth factor- $\beta$ (a key component of wound healing after glaucoma surgery ${ }^{34}$ ) were reduced in eyes after Ex-PRESS implantation, as compared with trabeculectomy (Sampson EM, ARVO abstract \#52, 2005).

In the present study, trabeculectomy and Ex-PRESS outcomes were evaluated in a prospective, randomised study where each patient underwent each procedure in a different eye. Although good IOP control was achieved in both groups, mean IOP values were marginally lower from 3 months to 2 years after Ex-PRESS implantation. Furthermore, success rates were higher throughout 30 months of follow-up after Ex-PRESS. Complete success rates are probably more relevant than qualified success rates because of compliance issues, especially in 
our study, which included only POAG patients with advanced optic nerve damage. In addition, trabeculectomy resulted in a higher rate of postoperative complications (33\%) than Ex-PRESS implants (20\%), and $27 \%$ of eyes needed postoperative interventions, whereas none were needed with Ex-PRESS. These results were obtained by a single experienced glaucoma surgeon (ED) who has performed several thousands of trabeculectomies since 1981 and initiated the Ex-PRESS implantation under a scleral flap in the year 2000.

Trabeculectomy eyes had higher rates of hypotony during the immediate postoperative period than Ex-PRESS eyes. The difference in hypotony rates can be explained by the essential difference in the immediate postoperative filtration mechanism of the two procedures. The physical properties of the apertures that connect the anterior chamber to the sub-scleral space are not same in the two procedures. The aqueous flow through Ex-PRESS, which is essentially a 2.4-mm-long tube with a $200-\mu \mathrm{m}$ lumen, is probably different from the flow through the $1.5-\mathrm{mm}^{2}$-wide sclerectomy of the trabeculectomy even when both apertures are secured by overlying scleral flaps. Although the scleral flaps were closed with the same numbers of sutures in both groups, there is a possibility that the higher hypotony rate in the trabeculectomy group was due to occasional differences in suture tension between the two techniques.

When trabeculectomy and Ex-PRESS implantation are compared, the cost issue of the latter cannot be ignored especially in a public healthcare environment. The two procedures resulted in similar postoperative IOPs but implantation of a foreign device implies additional cost. However, the cost of Ex-PRESS might be outweighed by its benefits in terms of lower complication rates, lower postoperative intervention rates, and above all, its higher complete success rate that relieves patients from the costs of postoperative anti-glaucoma medications. It is up to the surgeon to judge when to prefer one procedure over the other according the economic situation, availability of implant and his/her judgment on the cost of postoperative anti-glaucoma medications, the hidden costs of complications, and the need for postoperative additional interventions.

Our results are in accordance with those of a prospective, comparative, randomised study by De Jong during a follow-up limited to 1 year. ${ }^{18}$ Complete success rate was $81.8 \%$ after Ex-PRESS compared with $47.5 \%$ after trabeculectomy at a 1-year follow-up. The need for postoperative interventions was 15\% after trabeculectomy and 7.5\% after Ex-PRESS.

Gallego-Pinazo et al ${ }^{15}$ compared Ex-PRESS implantation with trabeculectomy, in combined cataract/ filtering operations with a mean follow-up of 9.7 months. They reported a significantly higher complication rate during the early postoperative course after trabeculectomy than after Ex-PRESS implantation. IOP reduction was similar in both groups, but IOP control was better after Ex-PRESS implantation, although not statistically significant. Our study yielded comparable results during a longer follow-up.

Maris $e t a l^{14}$ performed a retrospective comparison of trabeculectomy vs Ex-PRESS implantation. As in the present study, these investigators also noted a higher rate of postoperative hypotony after trabeculectomy (32\%) compared with Ex-PRESS implantation (4\%). Unlike us, however, their Kaplan-Meier analysis showed no significant difference between success rates or the need for supplemental glaucoma medication. The differences between their study and ours may be partly explained by the various glaucoma types included in their study such as neovascular and uveitic glaucoma that are known to have different natural histories and worse prognoses than POAG. Furthermore, the upper IOP cut-off point for 'success' in their study was set at $21 \mathrm{~mm} \mathrm{Hg}$, whereas in our study it was set at $<18 \mathrm{~mm} \mathrm{Hg}$. We chose this relatively low IOP cut-off point for 'success' because all our patients' eyes manifested advanced POAG with cup disk ratio $\geq 0.85$, where a low target postoperative IOP is desirable.

Good and Kahook ${ }^{20}$ reported a retrospective comparison between 35 consecutive Ex-PRESS implants and 35 consecutive trabeculectomy procedures followed up for at least 2 years. Ex-PRESS implantation yielded a $77.14 \%$ complete success rate, compared with $74.29 \%$ with trabeculectomy. Blebs with Ex-PRESS were fewer, less vascular, and more diffuse than after trabeculectomy (Moorfields Bleb Grading System), and cases of early postoperative hypotony and hyphema were fewer. Visual recovery was more rapid in eyes with Ex-PRESS. The different complete success rates, between their study and ours, may be partly explained by their inclusion of patients with chronic angle-closure glaucoma and pseudoexfoliation glaucoma, in addition to POAG. Furthermore, these two studies were retrospective and procedures were not contrasted between eyes of individual patients, as in the present report.

Severe postoperative complications were uncommon after either intervention in our study, where the standardised technique for both interventions included systematic use of an ACM. This permitted consistent control of positive intra-operative IOP, and enabled us to assess and modulate the filtration, to achieve a mild aqueous percolation at the posterior edges of the scleral flap after the scleral flap had been sutured. We also constructed a relatively large scleral flap $(5 \times 5 \times 1 \mathrm{~mm})$ to increase the area for dispersing the aqueous flow. These aspects of our technique were previously described as features conducive to safer and more 
effective surgical outcomes. ${ }^{35}$ Several investigators have noted a risk of central visual acuity loss after filtration surgery in eyes with advanced glaucoma. ${ }^{36,37}$ We did not encounter this phenomenon in any eye in the present study, which seems particularly striking as all eyes studied had advanced POAG with a mean pre-operative cup disk ratio $\geq 0.85$. It may be that our adoption of the 'safe trabeculectomy' technique, ${ }^{35,38}$ with its wide scleral flap and systematic use of an ACM, was beneficial in all cases.

The major weakness of our study is its small sample size, only 30 eyes studied (15 patients), due to the relative difficulty in enrolling POAG patients who agree to undergo trabeculectomy in one eye and Ex-PRESS implantation in the other eye in a randomised manner. However, this small group was sufficient to show a statistically significant difference between procedures especially on criteria of success rates and complication rate. The validity of our findings was further strengthened by comparing the two interventions in fellow eyes of the same patient. At last, all patients had advanced POAG in both eyes, and therefore presented a uniform and homogeneous group appropriate for a well-founded comparison of the two interventions.

A lesser limitation regarding lack of masking still exists in our study. It was not possible to mask the surgical technique as trabeculectomy is easily differentiated from Ex-PRESS implantation during postoperative follow-ups. However, this limitation is overcome by the fact that all patients were followed up concurrently by their referring ophthalmologists from the first month postoperatively till completion of the study.

In summary, the present study is the first report of a prospective, randomised comparison of trabeculectomy and Ex-PRESS implantation in fellow eyes of the same patient with the longest follow-up period to date. Our results are consistent with other published reports showing that the Ex-PRESS device provides similar IOP control to trabeculectomy, with lower incidence of postoperative complications, additional interventions, or need for glaucoma medications.

\section{Summary}

\section{What was known before}

- Ex-PRESS provides IOP control similar to trabeculectomy. Ex-PRESS has less postoperative complications than trabeculectomy.

\section{What this study adds}

- Glaucoma patients after Ex-PRESS implantation need less postoperative interventions than after trabeculectomy. The complete success rates are higher with Ex-PRESS than with trabeculectomy.

\section{Conflict of interest}

ED is a paid consultant to Alcon Laboratories. GJBS has no financial or proprietary interest in any of the drugs or materials mentioned in this study. AL has no financial or proprietary interest in any of the drugs or materials mentioned in this study. AL is employed by CEMKAEVAL, a company that provides services in statistical analyses and epidemiology.

\section{Acknowledgements}

The study was supported by a financial grant from Alcon Laboratories. We thank Corinne Emery, Babak Khoshnood, and Stephane Bouee for assistance in data management and statistical analysis, and Robert Pigache, MD, for assistance with the writing of the paper.

\section{References}

1 Borisuth NS, Phillips B, Krupin T. The risk profile of glaucoma filtration surgery. Curr Opin Ophthalmol 1999; 10: 112-116.

2 Gedde SJ, Herndon LW, Brandt JD, Budenz DL, Feuer WJ, Schiffman JC. Surgical complications in the Tube Versus Trabeculectomy Study during the first year of follow-up. Am J Ophthalmol 2007; 143: 23-31.

3 Gedde SJ, Schiffman JC, Feuer WJ, Herndon LW, Brandt JD, Budenz DL. Treatment outcomes in the Tube Versus Trabeculectomy study after one year of follow-up. Am J Ophthalmol 2007; 143: 9-22.

4 Sarodia U, Shaarawy T, Barton K. Non penetrating glaucoma surgery: a critical evaluation. Curr Opin Ophthalmol 2007; 18: 152-158.

5 Carassa RG, Bettin P, Fiori M, Brancato R. Viscocanalostomy versus trabeculectomy in White adults affected by open angle glaucoma: a 2 year randomized, controlled trial. Ophthalmology 2003; 110: 882-887.

6 Wamsley S, Moster MR, Rai S, Alvim HS, Fontanarosa J. Results of the use of the Ex-PRESS miniature glaucoma implant in technically challenging, advanced glaucoma cases: a clinical pilot study. Am J Ophthalmol 2004; 138: 1049-1051.

7 Garg SJ, Kanitkar K, Weichel E, Fischer D. Trauma-induced extrusion of an Ex-PRESS glaucoma shunt presenting as an intraocular foreign body. Arch Ophthalmol 2005; 123: 1270-1272.

8 Stewart RM, Diamond JG, Ashmore ED, Ayyala RS. Complications following Ex-PRESS glaucoma shunt implantation. Am J Ophthalmol 2005; 140: 340-341.

9 Traverso CE, De Feo F, Messas-Kaplan A, Denis P, Levartovsky S, Sellem E et al. Long term effect on IOP of a stainless steel glaucoma drainage implant (Ex-PRESS) in combined surgery with phacoemulsification. Br J Ophthalmol 2005; 89: 425-429.

10 Tavolato M, Babighian S, Galan A. Spontaneous extrusion of a stainless steel glaucoma drainage implant (Ex-PRESS). Eur J Ophthalmol 2006; 16: 753-755.

11 Stein JD, Herndon LW, Brent Bond J, Challa P. Exposure of Ex-PRESS Miniature Glaucoma Devices: case series and 
technique for tube shunt removal. J Glaucoma 2007; 16: 704-706.

12 Rivier D, Roy S, Mermoud A. Ex-PRESS R-50 miniature glaucoma implant insertion under the conjunctiva combined with cataract extraction. J Cataract Refract Surg 2007; 33: 1946-1952.

13 Dahan E, Carmichael TR. Implantation of a miniature glaucoma device under a scleral flap. J Glaucoma 2005; 14: 98-102.

14 Maris Jr PJ, Ishida K, Netland PA. Comparison of trabeculectomy with Ex-PRESS miniature glaucoma device implanted under scleral flap. J Glaucoma 2007; 16: 14-19.

15 Gallego-Pinazo R, López-Sánchez E, Marín-Montiel J. Postoperative outcomes after combined glaucoma surgery. Comparison of Ex-PRESS miniature implant with standard trabeculectomy. Arch Soc Esp Oftalmol 2009; 84: 293-298.

16 Coupin A, Li Q, Riss I. Ex-PRESS miniature glaucoma implant inserted under a scleral flap in open-angle glaucoma surgery: a retrospective study. J Fr Ophtalmol 2007; 30: 18-23.

17 Kanner EM, Netland PA, Sarkisian SR, Du H. Ex-PRESS miniature glaucoma device implanted under a scleral flap alone or combined with phacoemulsification cataract surgery. J Glaucoma 2009; 18: 488-491.

18 De Jong LA. The Ex-PRESS glaucoma shunt versus trabeculectomy in open-angle glaucoma: a prospective randomized study. Adv Ther 2009; 26: 336-345.

19 De Feo F, Bagnis A, Bricola G, Scotto R, Traverso CE. Efficacy and safety of a steel drainage device implanted under a scleral flap. Can J Ophthalmol 2009; 44: 452-462.

20 Good TJ, Kahook MY. Assessment of bleb morphologic features and postoperative outcomes after Ex-PRESS drainage device implantation versus trabeculectomy. Am J Ophthalmol 2011; 151: 507-513.e1.

21 Zeger SL, Liang KY. Longitudinal data analysis for discrete and continuous outcomes. Biometrics 1986; 42: 121-130.

22 Royston P, Altman DG. Regression using fractional polynomials of continuous covariates: parsimonious parametric modelling. Appl Stat 1994; 43: 429-467.

23 Royston P, Ambler G, Sauerbrei W. The use of fractional polynomials to model continuous risk variables in epidemiology. Int J Epidemiol 1999; 28: 964-974.
24 Therneau T, Grambsch P. Modeling Survival Data Extending the Cox Model. Springer: New York, NY, USA, 2000.

25 Therneau T. Survival: survival analysis, including penalised likelihood. R package version 2.35-8; http:/ /CRAN. R-project.org/package $=$ survival, 2009.

26 R Development Core Team. R: a Language and Environment for Statistical Computing. R Foundation for Statistical Computing: Vienna, Austria. ISBN 3-900051-07-0; URL:http:/ / www.R-project.org, 2010.

27 Katz LJ. A call for innovative operations for glaucoma. Arch Ophthalmol 2000; 118: 412-413.

28 Minckler DS, Baerveldt G, Alfaro MR, Francis BA. Clinical results with the Trabectome for treatment of open-angle glaucoma. Ophthalmology 2005; 112: 962-967.

29 Schwartz KS, Lee RK, Gedde SJ. Glaucoma drainage implants: a critical comparison of types. Curr Opin Ophthalmol 2006; 17: 181-189.

30 Nyska A, Glovinsky Y, Belkin M, Epstein Y. Biocompatibility of the Ex-PRESS miniature glaucoma drainage implant. J Glaucoma 2003; 12: 275-280.

31 De Feo F, Jacobson S, Nyska A, Pagani P, Traverso CE. Histological biocompatibility of a stainless steel miniature glaucoma drainage device in humans: a case report. Toxicol Pathol 2009; 37: 512-516.

32 Dahan E. Ex-PRESS miniature glaucoma implant. Expert Rev Ophthalmol 2007; 2(6): 899-909.

33 Hendrick AM, Kahook MY. Ex-PRESS min glaucoma shunt: surgical technique and review of clinical experience. Expert Rev Med Devices 2008; 5: 673-678.

34 Cordeiro MF. Role of transforming growth factor beta in conjunctival scarring. Clin Sci (Lond) 2003; 104: 181-187.

35 Jones E, Clarke J, Khaw PT. Recent advances in trabeculectomy technique. Curr Opin Ophthalmol 2005; 16: 107-113.

36 Costa VP, Smith M, Spaeth GL, Gandham S, Markovitz B. Loss of visual acuity after trabeculectomy. Ophthalmology 1993; 100: 599-612.

37 Levene RZ. Central visual field, visual acuity, and sudden visual loss after glaucoma surgery. Ophthalmic Surg 1992; 23: 388-394.

38 Stalmans I, Gillis A, Lafaut AS, Zeyen T. Safe trabeculectomy technique: long term outcome. $\mathrm{Br}$ J Ophthalmol 2006; 90: 44-47. 